\title{
AMCoR
}

Asahikawa Medical University Repository http://amcor.asahikawa-med.ac.jp/

Colloids Surf B Biointerfaces. (2014) 122:1-6.

Wetting dynamics of colloidal dispersions on agar gel surfaces.

Seino E, Chida S, Mayama H, Hotta J, Nonomura Y. 


\section{Wetting dynamics of colloidal dispersions on agar gel surfaces}

Eri Seino ${ }^{\mathrm{a}}$, Shigeki Chida ${ }^{\mathrm{a}}$, Hiroyuki Mayama ${ }^{\mathrm{b}}$, Jun-ichi Hotta ${ }^{\mathrm{c}}$, and Yoshimune Nonomura $^{\mathrm{a} *}$

a Department of Biochemical Engineering, Graduate School of Science and Engineering Yamagata University, 4-3-16 Jonan, Yonezawa 992-8510

${ }^{\mathrm{b}}$ Research Institute for Electronic Science, Hokkaido University, CRIS Building, N21W10, Sapporo 001-0021, Japan Present address: Department of Chemistry, Asahikawa Medical University, 2-1-1-1 Midorigoka-Higashi, Asahikawa, Hokkaido 078-8510, Japan

${ }^{\mathrm{c}}$ Department of Bio-systems Engineering, Graduate School of Science and Engineering Yamagata University, 4-3-16 Jonan, Yonezawa 992-8510

${ }^{*}$ Corresponding author. Tel.: $+84-238-26-3164$.

E-mail address: nonoy@yz.yamagata-u.ac.jp (Y. Nonomura)

Statistical summary:

The total number of words $=4700$

The total number of tables $=1$

The total number of figures $=5$ 


\section{ABSTRACT}

We have analyzed the effects of the addition of silica particles on the wetting velocity at flat and fractal agar gel surfaces and the applicability of such particles for controlling the wetting dynamics of water. We found that the contact angle $\left(\theta_{D}\right)$ of colloidal dispersions followed the power law $\theta_{D} \propto t^{-x}$, where $t$ and $x$ are time and a constant. The wetting is inhibited by the addition of a suitable amount of silica particles with a diameter of $20 \mathrm{~nm}$. Specifically, the lowest value for the exponent $x$ occurred when the silica composition was $0.1 \mathrm{wt} \%$. However, such inhibition effects were not observed on the addition of silica particles with a diameter of 100,550 and $1000 \mathrm{~nm}$. The mechanism of inhibition of the wetting of liquids on gel surfaces can be attributed to slight increase in the local viscosity around the contact line during wetting.

Keywords:

Wetting phenomena

Colloidal dispersion

Crystal structure

Agar gel 


\section{Introduction}

Wetting dynamics of liquids on biological interfaces are one of the most crucial mass transport phenomena in the human body because these processes contribute to various biological functions, including nutritional absorption at the wall of the small intestine, the occurrence of dry eye syndrome on the corneal surface, and sensitization of the tongue surface [1-3]. Most biological interfaces are complex systems with hierarchical roughness and are generally coated with mucus. Therefore, there are only a few reports exploring the control of wetting dynamics on biological interfaces owing to difficulties in performing direct observations or physical evaluations [4-6]. Recently, we have succeeded in preparing agar gels with rough surfaces, referred to as "fractal agar gels," as models of biological surfaces [7]. The rough structure of the fractal agar gels mimics the hierarchical structure of the walls of the small intestine, which consist of circular folds, villuses, and microvilluses. We evaluated the wetting velocity of water on fractal gel surfaces quantitatively by a high speed camera and confirmed that the wetting of water was accelerated by the ultra-rough structure on the gel surfaces. A theoretical model of wetting behavior on rough surfaces was proposed on the basis of the changes in free energy associated with wetting phenomena [8].

To acquire a deep understanding of the wetting dynamics, it is crucial to not only observe the wetting process but also find experimental and theoretical strategies to control it. In general, the wetting dynamics of a liquid depend on the interfacial tension and viscosity of the liquid [9]. Hoffman showed that the characteristic moving velocity of three-phase contact lines can be described by $\gamma \eta \eta$, where $\gamma$ and $\eta$ are the surface tension and viscosity of the liquid, respectively [10]. In our previous study, we observed 
anomalous wetting behavior for aqueous alcohol solutions on agar gel surfaces [11]. The extremely fast wetting was caused by strong Marangoni flow at the three-phase contact line.

We focus herein on rheology effects for spreading dynamics. Rafai et al. studied the spreading of droplets of complex fluids having either surfactants or polymers dissolved in the fluid [Rafai et al. studied this: Spreading of non-Newtonian fluids on hydrophilic surfaces Journal of Fluid Mechanics 513 (1), 77-85 (2004); Spreading of non-Newtonian fluids and surfactant solutions on solid surfaces Physica A: Statistical Mechanics and its Applications 358 (1), 58-67 (2005)]. It turns out that both surfactants and polymers slow down the spreading. A special type of surfactants (trisiloxanes), however, leads to a " superspreading" , in which the droplet spreads out orders of magnitude quicker than with usual surfactants. The addition of colloidal particles might diminish the wetting of liquids owing to the formation of network structures and induction of thickening effects by the dispersed solid particles. Rio et al. showed that a stick-slip phenomenon and pinning effect occur as a result of aggregation of particles at the three-phase contact line when a colloidal dispersion come in contact with a glass substrate [12]. Furthermore, a solid-like ordering of suspended spheres occurs in the confined three-phase contact region at the edge of the wetting fluid, as described by Wassan et al. who used video microscopy to demonstrate the two-dimensional crystal-like ordering of charged nanometer-sized polystyrene spheres in water $[13,14]$. Koh and Wong utilized reflectance spectroscopy to monitor structural changes during the self-assembly of a monodisperse colloidal system at the three-phase contact line of a sessile drop on an inert substrate [15]. Solids dispersed in a drying 
drop will migrate to the edge of the drop and form a solid ring called a "coffee ring." The migration is caused by an outward flow within the drop that is driven by the loss of solvent (through evaporation) and geometrical constraints requiring that the drop maintain an equilibrium droplet shape with a fixed boundary [16-19]. The dewetting causes the formation of finger-like patterns near the contact line which leave behind a deposit of branches. We find that the strikingly low speed of dewetting is due to the high particle concentration in the contact line region, leading to a strongly enhanced viscosity. For pattern formation from drying droplets (containing particles), evaporation also causes dewetting. [M. Habibi, P. Moller, A. Fall, S. Rafaid, D. Bonn, Soft Matter, $2012,8,4682-4686$.] When the particle concentration is above a threshold value, the size can be estimated by considering the collective effects of droplet evaporation and diffusive motion of the particle within the droplet.

In this study, we observed the wetting behavior of silica particle dispersions $(20$, 100,550 and $1000 \mathrm{~nm}$ particle sizes) on gel surfaces by a high speed camera to clarify whether the addition of colloidal particles affects the dynamics of liquid on agar gel surfaces. We also investigated the wetting behavior of liquids on fractal gel surfaces with hierarchical roughness to clarify the effects of colloid size or rough hierarchical structure of the gel surface on the wetting velocity. Finally, we suggested a phenomenological physical model on the basis of the experimental findings to describe the wetting behavior of colloidal dispersions. Fractal agar gel is suitable for the model of these biological surfaces because it is a hydrophilic surface with hierarchical roughness. Silica nanoparticles are suitable because of their low toxicity: the control technology of wetting dynamics is useful for skin care products, cleansing products, and 
so on. For example, inhibition agents of wetting can be applicable to control drug delivery efficiency in future.

\section{Experimental}

\subsection{Materials}

Agar powder was purchased from Kanto Chemical Co. (Tokyo, Japan) and used without further purification. The two types of silica particles, Aerosil 200 (amorphous shape, mean particle diameter: $20 \mathrm{~nm}$ ) and COSMO55 (spherical shape, mean particle diameter: $550 \mathrm{~nm}$ ), were obtained from Nippon Aerosil Co., Ltd. (Tokyo, Japan) and JGC Catalysts and Chemicals Ltd. (Kanagawa, Japan), respectively. Two types of silica particle dispersions, sicastar 43-00-102 (100 $\mathrm{nm}$ in diameter, $\left.50 \mathrm{mg} \mathrm{mL}^{-1}\right)$ and sicastar 43-00-103 (1000 $\mathrm{nm}$ in diameter, $50 \mathrm{mg} \mathrm{mL}^{-1}$ ), and a fluorescent-particle dispersion, sicastar green F 42-00-301 (30 nm in diameter, $50 \mathrm{mg} \mathrm{mL}$ ), were purchased from Nacalai Tesque, Inc. (Kyoto, Japan).

\subsection{Preparation}

Flat and fractal agar gels were prepared by the method described in a previous study [7]. Briefly, to prepare the flat agar gel, a mixture of agar powder $(6 \mathrm{~g})$ and deionized water (144 g) was heated and agitated until the agar powder dissolved. We then poured $150 \mathrm{~g}$ of the $4 \mathrm{wt} \%$ agar aqueous solution into Petri dishes $9 \mathrm{~cm}$ in diameter after which the solution was allowed to cool and solidify. Fractal agar gels were also prepared by a previously reported method $[7,11]$. The templates for the fractal surfaces were prepared by the solidification of melted alkylketene dimer (AKD). A plaster 
replica was prepared using the AKD surface as a mold. Agar aqueous solution was poured into Petri dishes containing the plaster replica. Box counting measurements confirmed the surface structures to be fractals with fractal dimension $D=2.2$. Self-similarity was found to hold between box size 4 and $30 \mu \mathrm{m}$. The geometry of agar gel surfaces were observed by a VK-X100 laser microscope (Keyence, Tokyo, Japan). The roughness was as follows: flat agar gel $R_{\mathrm{a}}=0.026 \mu \mathrm{m}, R_{\mathrm{z}}=0.316 \mu \mathrm{m}, R_{\mathrm{y}}=0.430$ $\mu \mathrm{m}$, fractal agar gel $R_{\mathrm{a}}=2.14 \mu \mathrm{m}, R_{\mathrm{z}}=31.7 \mu \mathrm{m}, R_{\mathrm{y}}=35.7 \mu \mathrm{m}$. Silica particles were mixed with water purified by a water deionizing unit (DX-15 from Kurita Water Industries Ltd., Tokyo, Japan) for $30 \mathrm{~s}$ in a screw-cap test tube using a vortex mixer (Vortex-genie 2, Scientific Industries Inc., New York, USA) and then remixed using an NR-50M handheld ultrasonic homogenizer (Microtec Co., Ltd., Chiba, Japan) for $30 \mathrm{~s}$ to obtain silica particle dispersions.

\subsection{Measurements}

The contact angle of the liquids on the gel surfaces was estimated on the basis of a sessile drop measuring method using a DM-501 contact angle meter (Kyowa Interface Science, Saitama, Japan) for a drop volume of $0.5 \mu \mathrm{L}$. The measurements were conducted in air at $298 \mathrm{~K}$ and $50 \%$ humidity. The liquid droplets were dropped by an automatic dispenser designed for the DM-501 contact angle meter from the tip of a $22 \mathrm{G}$ syringe (internal diameter: $0.4 \mathrm{~mm}$, external diameter: $0.7 \mathrm{~mm}$ ) coated with tetrafluoroethylene. The magnification of the images was about 32 when we observed them through a 17-inch display. Resolution of the display was $20 \mu \mathrm{m}$ of one pixel. The measurement of contact angle below $20^{\circ}$ is normally difficult, therefore, we verified the accuracy of the value. The accuracy of present method was within $1.6^{\circ}$ at 
$6.7^{\circ}$, for example (see Supporting information Table S1 and S2) [20]. The temporal change of contact angles at intervals of $1 \mathrm{~ms}$ were measured for ten different points and were averaged. We checked the initial volume of the colloidal dispersions on the flat and fractal agar gel surfaces. The volume was derived from the radius $(r)$ and height $(h)$ using the equation $V=(\pi / 3) h^{2}(3 r-h)$. The averages of the apparent initial volumes on the flat and fractal agar gel surfaces were $0.28-0.43$ and $0.29-0.47 \mu \mathrm{L}$, respectively. The liquid volume decreased by several tens percent within $1 \mathrm{~s}$ of instillation due to evaporation. However, the variability in the initial volume and the temporal changes had a limited effect on the wetting dynamics of the liquids: assuming a power law for $\theta_{D}(t)$ of the form $\theta_{D}(t) \propto t^{-x}$, the exponent $x$ was constant at initial volumes in the range of $0.3-0.7 \mu \mathrm{L}$. We investigated the influence of volume changes of the wetting droplet due to evaporation on the time course of the contact angle changes during wetting. From this evaluation, we confirmed that the changes in the contact angle can be neglected, at least within $1 \mathrm{~s}$ of instillation [11]. The surface tension of the liquids was estimated on the basis of the Young Laplace method using the DM-501 contact angle meter for the water drop volume of $10 \mu \mathrm{L}$. The surface tension values of the colloidal dispersions were shown in Table S3 in supporting information. The viscosity of the dispersions at $298 \mathrm{~K}$ was determined from their kinetic viscosity and density. The kinetic viscosity was measured using a VM-1A-L vibratory viscometer (Yamaichi Electronics Co., Ltd., Tokyo, Japan). The density was measured using a pycnometer. The viscosity values of the colloidal dispersions were shown in Table S2 in supporting information. The amounts of particles or solution are estimated by fluorescence intensity during wetting on ager. An inverted fluorescence microscope (IX71, Olympus) equipped with a $100 \mathrm{~W}$ 
mercury lamp (U-LH100HGAPO, Olympus) was used for fluorescence imaging. A 460-490 nm bandpass filter (BP460-495) was used for excitation and a $510 \mathrm{~nm}$ barrier filter (BA510IF) was used to select fluorescence of fluorescent silica beads and rhodamine B. Objective lenses (UPlanFLN4X and LUCPlanFLN40X, Olympus, Japan) were used, and a CMOS camera was used for fluorescence image recording. A sample gel was placed on a cover slide $(24 \mathrm{~mm} \times 60 \mathrm{~mm}$, Thickness No.1, Matsunami Glass Ind., Ltd., Japan). We used the sample volume of $1 \mu \mathrm{L}$ for fluorescence imaging.

\section{Results}

\subsection{Wetting dynamics of colloidal dispersions on agar gel surfaces}

When droplets of water and a $0.1 \mathrm{wt} \%$ colloidal dispersion containing silica particles $20 \mathrm{~nm}$ in diameter came in contact with flat agar gel surfaces, the droplets spread along the surfaces as shown in Figure 1. Briefly thereafter (for example, at $t=30$ $\mathrm{ms}$ ), we did not find significant differences in the wetting dynamics between water and the $0.1 \mathrm{wt} \%$ colloidal dispersion; the wetting droplet took the form of a hemi-spherical cap with a small dynamic contact angle. However, when $t$ reached 300 and $1000 \mathrm{~ms}$, the $0.1 \mathrm{wt} \%$ colloidal dispersion formed a thicker hemi-spherical cap with a larger contact angle than water. To quantify the effects of these wetting properties, the time-dependent variation of the contact angle $\left(\theta_{D}\right)$ during the dynamic wetting process was measured. Figure 2 shows the time course for $\theta_{D}$ of water and that of the two $0.1 \mathrm{wt} \%$ colloidal dispersions containing silica particles of diameters 20 and $550 \mathrm{~nm}$. In all cases, the contact angle decreased during the wetting process. These patterns of decline were linear on $\log -\log$ plots and followed the power law. For all conditions, the fit using 
power law was obtained by the method of least squares: the correlation coefficients between experimental data and fitting data were $0.90-0.97$. These results roughly follow Tanner's law for smooth and clean surfaces in which $\theta_{D}$ is proportional to $t^{-0.3}$; for example, in the case of water, $\theta_{D}$ was proportional to $t^{-0.27}$ for $0-4000 \mathrm{~ms}$. Interestingly, a turning point was observed on the contact angle profile only for the colloidal dispersion containing silica particles $20 \mathrm{~nm}$ in diameter. In this case, $\theta_{D}$ was proportional to $t^{-0.20}$ and $t^{-0.08}$ during the first period $(0-300 \mathrm{~ms})$ and the second period (300-4000 ms), respectively. On the other hand, in the case of the silica dispersion containing particles $550 \mathrm{~nm}$ in diameter, $\theta_{D}$ was proportional to $t^{-0.24}$ during the second period. We found that the wetting of liquids was drastically inhibited on agar gel surfaces by the addition of silica particles with suitable diameter.

\subsection{Effects of the composition of the colloidal dispersions.}

We examined the effects of the composition of the colloidal dispersions on flat agar gel surfaces. Figure $2 \mathrm{~b}$ shows the exponent $x$ assuming a power law of $\theta_{D} \propto t^{-x}$ in the first period of 0-300 ms. For all dispersions in which the colloidal composition was $0-4 \mathrm{wt} \%, x$ was almost constant $(\sim 0.3)$. Figure $2 \mathrm{c}$ shows the wetting velocity $(x)$ in the second period. In the case of colloidal dispersions containing colloidal silica particles 20 $\mathrm{nm}$ in diameter, the wetting velocity was inhibited as the concentration of particles increased. The exponent $x$ was minimum when the silica composition was equal to 0.1 $w t \%$. On the other hand, the inhibition effect was not observed for all dispersions containing particles of $100 \mathrm{~nm}$ and $550 \mathrm{~nm}$ diameter. In the cases of the dispersions containing particles of $1000 \mathrm{~nm}$ diameter, the inhibition was observed when the particle composition was more than $1 \mathrm{wt} \%$. We guess that this was caused by the damming effect of precipitated particles. These results suggest that the addition of colloidal 
particles of a suitable size and concentration are required to inhibit the wetting velocity.

\subsection{Effects of the rough structure of the fractal agar gels}

Figure $3 \mathrm{a}$ shows the temporal changes of the $\theta_{D}$ of water and the $0.1 \mathrm{wt} \%$ colloidal dispersions on the fractal agar gel surfaces. After the water droplets came in contact with the fractal agar gel surface $(3-500 \mathrm{~ms})$, the contact angle for water decreased from 34 to 3 degrees, and complete wetting was achieved. On the fractal agar gel, the decline pattern of the contact angle was linear on a log-log plot and followed the power law $\theta_{D} \propto t^{-0.52}$. It is clear that this fast wetting can be ascribed to the roughness of the fractal surface. When droplets of colloidal dispersions containing silica particles 20 or $550 \mathrm{~nm}$ in diameter were released onto the surface, the profiles of their $\theta_{D}$ values were in accord with that of water $\left(\theta_{\mathrm{D}}\right.$ was proportional to $t^{-0.49}$ or $\left.t^{-0.51}\right)$ : the wetting velocity as not inhibited by the addition of colloidal particles on the fractal surfaces. These results show that the relationship between the roughness of the surface and the size of the colloidal particles is an important factor which determines if the inhibition effect is observed or not: if the surface roughness is much larger than the particle size, the effect does not occur.

\section{Discussion}

\subsection{Wetting behavior of colloidal dispersions with thickening}

Regarding the mechanism of the inhibiting effect caused by the colloidal particles, attention is focused on the local condensation of such particles. As mentioned in the introduction, a solid-like ordering of the particles was observed in the confined three-phase contact region at the edge of the wetting fluid [13-15]. This condensation 
can induce thickening and inhibit wetting at the three-phase contact line. As mentioned in results (a), a turning point was observed on the contact angle profile only for the colloidal dispersion containing silica particles $20 \mathrm{~nm}$ in diameter: $\theta_{\mathrm{D}}$ was proportional to $t^{-0.20}$ and $t^{-0.08}$ during the first period $(0-300 \mathrm{~ms})$ and the second period $(300-4000$ $\mathrm{ms}$ ), respectively. Such inhibiting effect is common phenomena because we observed similar effect also on a glass substrate.

To demonstrate the local condensation mechanism, we examined wetting process of $0.1 \mathrm{wt} \%$ colloidal dispersion including green-fluorescent particles with $30 \mathrm{~nm}$ in diameter. On the flat agar gel surfaces, a green ring with several-tens $\mu \mathrm{m}$ in width was observed by a fluorescent microscope as shown in Figure 4a: the significant emission occurred only on the edge of the water droplet. On the other hand, in the case of rhodamine B aqueous solution, the fluorescent emission occurred from the central part of the droplet as well as the edge region (Figure 4b). Since the fluorescence intensities are proportional to the amount of fluorescent particles or rhodamine B solution, these results suggest that the colloidal particles with the several-tens $\mathrm{nm}$ in diameter are condensed at the three phase contact of a liquid droplet.

A simple scenario of the wetting behaviors with local thickening along the contact line can be conceptualized according to the guidance from Tanner's law. The essence of Tanner's law concerns changes in the contact angle under a constant droplet volume, energy dissipation, and the wetting force at the contact line. Thus, Tanner's law is derived from the rate of change in the contact angle as shown in Supporting information:

$$
\frac{d \theta_{D}}{d t}=-\frac{\gamma_{L}}{2 l L \eta} \theta_{D}^{13 / 3}
$$


where $\gamma_{L}$ is the surface tension of the liquid, $l$ is the size ratio in droplet and molecule size and $L$ is characteristic size of the droplet, respectively, and $\eta$ is the viscosity [21]. Usually, $\eta$ represents the viscosity of the bulk fluid, and it is equal to the local viscosity around the contact line. For the current systems, eq. (1) is valid if no aggregation occurs in wetting. However, in these cases, the local concentration of particles around the contact line is not constant, as thickening is experimentally suggested by the aggregation of the colloidal particles, where the aggregation results from the local condensation of particles and strong particle-particle interactions. Therefore, the effect of an increase in local viscosity should be considered. Theoretically, there are two ways to treat the thickening. One is the Einstein and extended equations, and the other applies the Matsumoto treatment [22]. In general, the former considers local viscosity around the colloidal particles as being due to surface charges, while the latter takes into account both the local viscosity and the particle-particle interactions. Here we adopt the latter because the particle-particle interactions resulting from the surface charges could not be neglected in the condensed zone around the contact line. Accordingly, the viscosity is described as

$$
\ln \left(\frac{\eta}{\eta_{0}}\right)=k(p) \phi+f m^{3} \phi^{2}
$$

where $\eta_{0}$ is the viscosity of fluid containing no particles, $\phi$ and $k(p)$ are the volume fraction of particles and a function of the aspect ratio $p$ of a particle, respectively, and $f$ and $m$ are a constant slightly depending on particle shape and a coefficient reflecting the particle-particle interaction, respectively. In general, the first term corresponds to the thickening according to the Einstein methodology, while the second term corresponds to two-body interactions. Assuming that the local concentration around the contact line 
increases with $t, \phi$ in eq. (2) can be expressed by

$$
\phi \sim t^{\varsigma}
$$

where $\zeta$ is an exponent reflecting information such as the average distance between individual or aggregated particles around the contact line. The thickening can be described in terms of the degree of polynomial based on Eq. (2):

$$
\ln \left(\frac{\eta}{\eta_{0}}\right)=\alpha t^{\varsigma}+\beta t^{2 \varsigma}
$$

where $\alpha=k(p)$ and $\beta=f m^{3}$. Using a simplified exponential form of eq. (4)

$$
\eta(t)=\eta_{0} \exp \left[\alpha t^{\varsigma}+\beta t^{2 \varsigma}\right]
$$

Eq. (1) is

$$
\frac{d \theta_{D}}{d t}=-\frac{\gamma_{L}}{2 l L \eta_{0}} \theta_{D}^{13 / 3} \exp \left[-\alpha t^{\varsigma}-\beta t^{2 \varsigma}\right]
$$

where the exponential component can be approximated at $t \ll 1$ as

$$
\frac{d \theta_{D}}{d t}=-\frac{\gamma_{L}}{2 l L \eta_{0}} \theta_{D}^{13 / 3}\left[1-\alpha t^{\varsigma}-\beta t^{2 \varsigma}\right]
$$

where $e^{-x} \sim 1-x$. From eqs. (6) and (7), we get

$$
\theta_{D}(t)=\theta_{0} t^{-0.3}\left(1-\frac{\alpha}{\varsigma+1} t^{\varsigma}-\frac{\beta}{2 \varsigma+1} t^{2 \varsigma}\right)^{-0.3}
$$

where $\theta_{0}=\left[3 \gamma_{L} /\left(20 l L \eta_{0}\right)\right]^{0.3}$. The fitting curve in Figure 5 is obtained using eq. (9), where $\theta_{0}=41.864, \alpha=0.1766, \beta=0.0300$, and $\zeta=0.2395$. According to Ref. $19, k(p)$ $=2.5$ and $f=0.027$ for spherical particles $(p=1), k(p)=216$ and $f=0.033$ for rod particles $(p=50)$ and $m \sim 10$. In comparison, the obtained $\alpha(=k(p))$ and $\beta\left(=f m^{3}\right)$ suggest that the silica particles are aggregated and the particle-particle interaction is weak because $k(p) \leq 1$ and $m \sim 1$ from $\alpha$ and $\beta$. Obviously, the curve reproduces the wetting behavior of the $20 \mathrm{~nm}$ colloidal suspension on flat agar gels under Tanner's law, while the very weak thickening is described by the expression $\phi \propto t^{0.24}$. Here, the effect 
of the volume change on the contact angle can be neglected within several seconds [11].

Next, we consider why the thickening effect is prominent in the $20 \mathrm{~nm}$ colloidal suspension on flat gels. One of the reasons is the number density of the particles. The density of the $20 \mathrm{~nm}$ colloidal suspension is approximately $2 \times 10^{4}$ times greater than that of the $550 \mathrm{~nm}$ colloidal suspension, which shows that local thickening readily occurs during the wetting of the $20 \mathrm{~nm}$ colloidal suspension. Another reason is related to the surface roughness. Obviously, a rough agar surface has numerous pockets or grooves that can capture the particles during wetting, while a flat surface does not disturb the aggregation of the colloidal particles. In other words, the particles are easily stuck on a rough surface, but not on a flat one. As a result, no thickening occurs during the wetting on fractal rough surfaces, and temporal changes in the contact angle are described by [7]

$$
\theta_{D}(t) \propto t^{-3(1+\varepsilon) /(10+\varepsilon)}
$$

where $\varepsilon$ is the velocity enhancement factor ( $\varepsilon=0$ for a flat surface, and $\varepsilon>0$ for a rough and fractal surface). Since wetting on the fractal rough surface follows the relationship $\theta_{D} \propto t^{-0.5}, \varepsilon=0.8$. We expect that $\varepsilon$ is correlated to the surface roughness and fractal dimensions. The physical meaning of $\varepsilon$ can be understood in terms of fluid velocity on a rough wall [23], but the correlation between $\varepsilon$ and the surface roughness with fractal dimensions will be addressed in a future study.

Wetting behaviors with several solution conditions were discussed above. Table 1 summarizes the wetting behaviors with and without thickening on flat and fractal rough agar surfaces. Consequently, the wetting behaviors can be summarized as

$$
\theta_{D}(t) \propto \Theta(t) t^{-3(1+\varepsilon) /(10+\varepsilon)} \theta_{D}(t) \propto \Theta(t) t^{-3(1+\varepsilon) /(10+\varepsilon)}
$$


where $\Theta(t)$ is a switching function related to thickening. If thickening occurs,

$$
\Theta(t)=\left(1-\frac{\alpha}{\varsigma+1} t^{\varsigma}-\frac{\beta}{2 \varsigma+1} t^{2 \varsigma}\right)^{-0.3}
$$

If no thickening occurs $(\zeta=0)$,

$$
\Theta(t)=1
$$

Our current results indicate that the wetting behavior of colloidal suspensions on flat surfaces is governed by aggregation, whereas on fractal surfaces wetting is nearly constant. In the previous study, we found that the wetting behavior on flat agar surfaces is also governed by Marangoni flow using alcohol solutions on flat surfaces $\left(\theta_{D} \sim t^{-0.75}\right)$, but the wetting behaviors of water and alcohol solutions with Marangoni flow on fractal agar gels are similar $\left(\theta_{D} \sim t^{-0.5}\right) .{ }^{11}$ In the case, we theoretically found that surface defects suppress the Marangoni effect. These findings for the wetting behavior on fractal rough surfaces directly indicate that the surface roughness of the small intestine can suppress the local condensation of micelles containing nutritional matter. In other words, surface roughness is essential for homogeneous adhesion of the micelles over the entire surface of the small intestine.

On the other hand, the mechanism of thickening could be explained by local aggregation of the particles around the contact line. It is well known that the withdrawing contact line in a drying droplet of colloidal suspensions shows pattern formations such as coffee ring, branch, and network structures [16-19]. In addition, the mechanisms of particle aggregation have been studied with regard to diffusion-limited aggregation (DLA) and cluster-cluster aggregation (CCA), although the simulations were carried out in the bulk phase [24-27]. The thickening around the contact line discussed here is observed in the intermediate stage between pattern formation and 
aggregation. Developing an understanding of the details of the thickening is an important consideration for future studies.

\section{Conclusions}

We found an inhibitory effect afforded by colloidal particles $20 \mathrm{~nm}$ in diameter on flat agar gel surfaces. On such surfaces, the power-law exponent $x$ for the relationship $\theta_{D}(t) \propto t^{-\mathrm{x}}$ decreased with wetting and attainted the minimum value of 0.08 for a $0.1 \mathrm{wt} \%$ dispersion, whereas $x$ was approximately 0.3 for the wetting of water and colloidal suspensions having particles of size $550 \mathrm{~nm}$. This inhibition effect is caused by thickening and the local condensation of colloidal particles at the three-phase contact line. However, on fractal gel surfaces, similar wetting behaviors $(x \sim 0.5)$ were observed for the wetting of water and all colloidal suspensions. The surface-dependent wetting is discussed in terms of thickening around the contact line and surface roughness. Aggregation occurs readily on flat surfaces, whereas it is inhibited by the grooves between the surface structures on fractal surfaces. This is comprehensively explained by energy dissipation under the slight increase in local viscosity around the contact line.

\section{Acknowledgment}

This research was supported by a Grant-in-Aid for Scientific Research on Innovative Area (No. 21106504 and 2310674) and Scientific Research (C) (No. 23540473) from the Ministry of Education, Culture, Sports, Science, and Technology, Japan (MEXT). 


\section{References}

[1] H. Schott, J. Pharm. Sci. 60 (1971) 1893.

[2] H. Ranc, A. Elkhyat, C. Servais, S. Mac-Mary, B. Launay, P. Humbert, Colloids Surf. A $276(2006) 155$.

[3] D. Hijrter, J. B. Dressman, Adv. Drug Delivery Rev. 25 (1997) 3.

[4] A. Farman, J. Ultrastruct. Res. 12 (1965) 328.

[5] N. Mygind, Rhinology13 (1975) 57.

[6] S. L. Palay, L. J. Karlin, J. Cell Biol. 5 (1959) 363.

[7] Y. Nonomura, Y. Morita, T. Hikima, E. Seino, S. Chida, H. Mayama, Langmuir 26 (2010) 16150.

[8] H. Mayama, Y. Nonomura, Langmuir 27 (2011) 3550.

[9] P.-G.de Gennes, Rev. Mod. Phys. 57 (1985) 827.

[10] R. Hoffman, J. Colloid Interface Sci. 50 (1975) 228.

[11] Y. Nonomura, S. Chida, E. Seino, H. Mayama, Langmuir 27 (2012) 3799.

[12] E. Rio, A. Daerr, F. Lequeux, L. Limat, Langmuir 22 (2006) 3186.

[13] D. T. Wasan, A. D. Nikolov, Nature 423 (2003) 156.

[14] O. K. Matar, R. V. Craster, K. Sefiane, Phys. Rev., 76 (2007) 056315.

[15] Y. K. Koh, C. C. Wong, Langmuir 22 (2006) 897.

[16] R. D. Deegan, O. Bakajin, T. F. Dupont, G. Huber, S. R.Nagel, T. A. Witten, Nature 389 (1997) 827.

[17] R. D. Deegan, O. Bakajin, T. F. Dupont, G. Huber, S. R. Nagel, T. A. Witten, Phys. Rev. E 62 (2000) 756.

[18] R. D. Deegan, Phys. Rev. E 61 (2000) 475. 
[19] W. Sempels, R. D. Dier, H. Mizuno, J. Hofkens, J. Vermant, Nat. Comm. 4 (2013) 1757.

[20] D. Seveno, A. Vaillant, R. Rioboo, H. Adão, J. Conti, J. D. Coninck, Langmuir 25 (2009) 13034.

[21] P. G. de Gennes, F. Brochard-Wyart, D. Quéré, Capillary and Wetting Phenomena: Drops, Bubbles, Pearls, Waves, Springer, New York, 2002, pp 142-150.

[22] S. Yao, T. Matsumoto, J. Non-Newtonian Fluid Mech. 25 (1987) 197.

[23] L. D. Landau, E. M. Lifshitz, Fluid Mechanics, 2nd ed. Butterworth-Heinemann, Boston, 1988.

[24] T. A. Witten, P. Meakin, Phys. Rev. B 28 (1983) 5632.

[25] P. Meakin, Phys. Rev. A 27 (1983) 604.

[26] D. A. Weitz, M. Oliveria, Phys. Rev. Lett. 52 (1984) 1433.

[27] T. Vicsek, Fractal Growth Phenomena 2nd. Ed., World Scientific, Singapore, 1992. 


\section{Figure Captions}

Figure 1. Droplets wetting on flat agar gel surfaces: (a) water and (b) $0.1 \mathrm{wt} \%$ colloidal dispersion containing silica particle with $20 \mathrm{~nm}$ in diameter. Scales $=0.5 \mathrm{~mm}$.

Figure 2. Wetting behavior of colloidal silica dispersions on flat agar gel surfaces. (a) Time course of the contact angle $\theta_{D}(t)$ for $0.1 \mathrm{wt} \%$ colloidal silica dispersions containing silica particles $20 \mathrm{~nm}(\square)$ and $550 \mathrm{~nm}(\square)$ in diameter and water ( $\square)$. (b) and (c) The relationship between the colloidal silica concentration and the exponent $x$ when $\theta_{D}(t) \propto t^{-x}: 0-300 \mathrm{~ms}(\mathrm{~b})$ and $300-4000 \mathrm{~ms}(\mathrm{c})$. The error bars are standard deviations of $\theta_{D}(t)$ and $x$, when contact angles were measured at ten different points and were averaged.

Figure 3. Wetting behavior of colloidal silica dispersions on fractal agar gel surfaces.

(a) Time course of the contact angle $\theta_{D}(t)$ of $0.1 \mathrm{wt} \%$ colloidal silica dispersions containing silica particles $20 \mathrm{~nm}(\square)$ and $550 \mathrm{~nm}(\square)$ in diameter and water ( $\square$ ). (b) The relationship between the colloidal silica concentration and the exponent $x$ when $\theta_{\mathrm{D}}(t) \propto t^{-x}$. The error bars are standard deviations of $\theta_{D}(t)$ and $x$, when contact angles were measured at ten different points and were averaged.

Figure 4. Fluorescence images of $0.1 \mathrm{wt} \%$ fluorescent-particle dispersion (a) and 0.01 $w t \%$ rhodamine B aqueous solution (b) on flat agar gel surfaces. 
Figure 5. Fitting results for the wetting behaviors of particles $20 \mathrm{~nm}$ (red curve) and $550 \mathrm{~nm}$ (blue curve) in diameter and water (black curve) by Tanner's law (thin solid green line) and eq. (8) (dashed black line).

Table 1. Wetting behaviors of the colloidal suspensions and water on flat and rough agar gel surfaces.
Case
Solution
Surface
Thickening
$\theta_{D}(t)$

$120 \mathrm{~nm}$ colloidal suspension

Flat $(\varepsilon=0)$

Yes

$t^{-0.08}$

$2 \quad 550 \mathrm{~nm}$ colloidal suspension

Flat $(\varepsilon=0)$

No

$t^{-0.23}$

3

water

Flat $(\varepsilon=0)$

No

$t^{-0.24}$

$4 \quad 20$ nm colloidal suspension

$\operatorname{Rough}(\varepsilon \neq 0) \quad$ No

$t^{-0.49}$

$550 \mathrm{~nm}$ colloidal suspension \& water

Rough $(\varepsilon \neq 0)$

No

$t^{-0.51}$

6

water

Rough $(\varepsilon \neq 0)$

No

$t^{-0.52}$ 


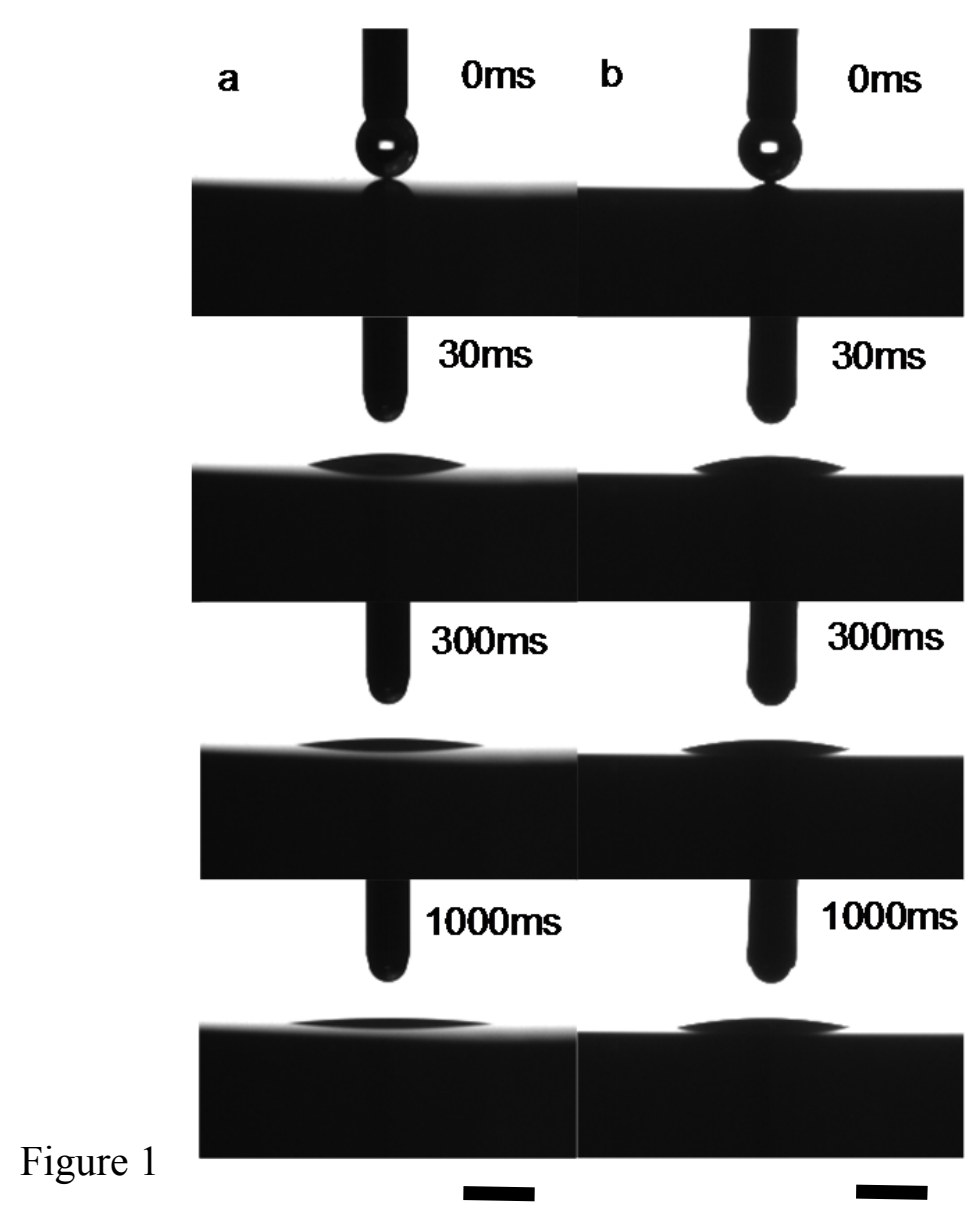

E. Seino et al 

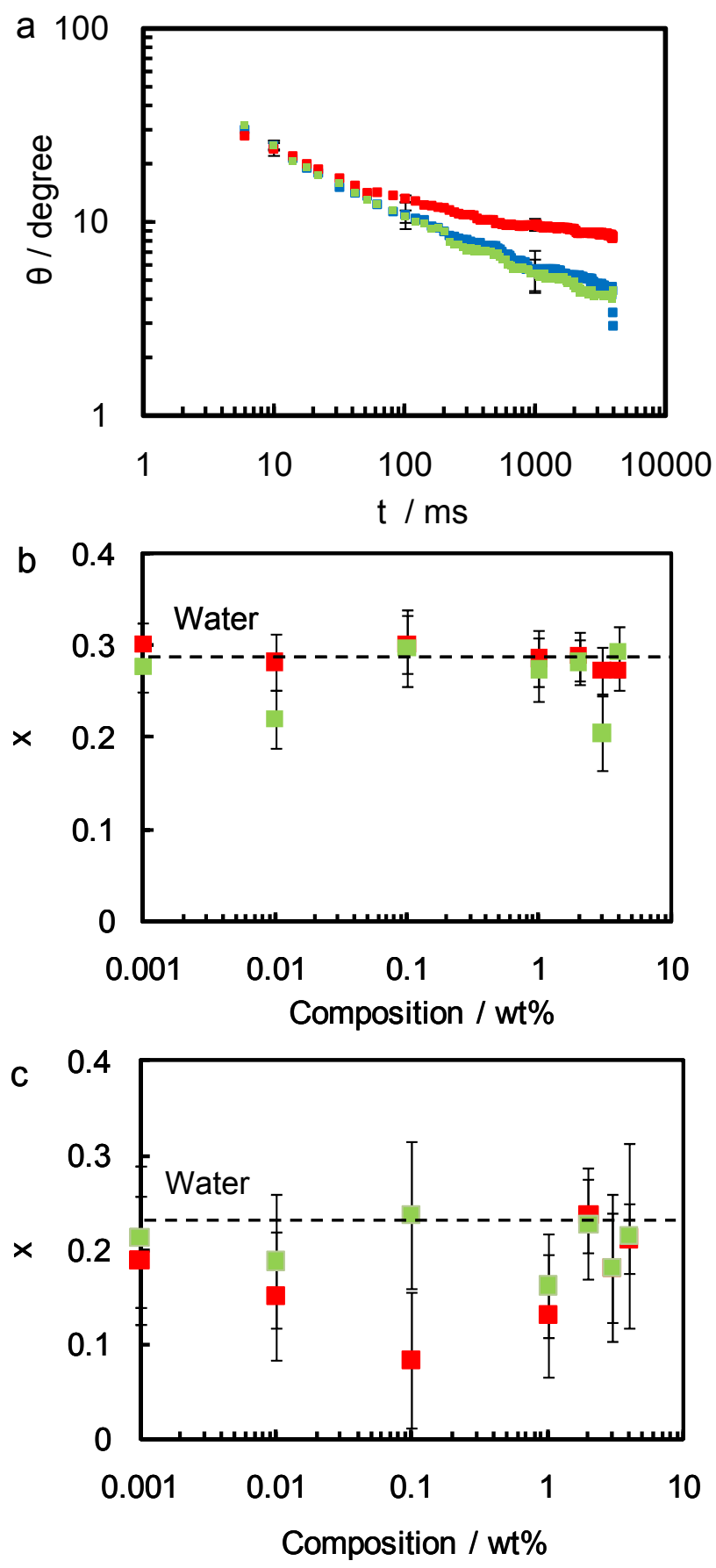

Figure 2

E. Seino et al 

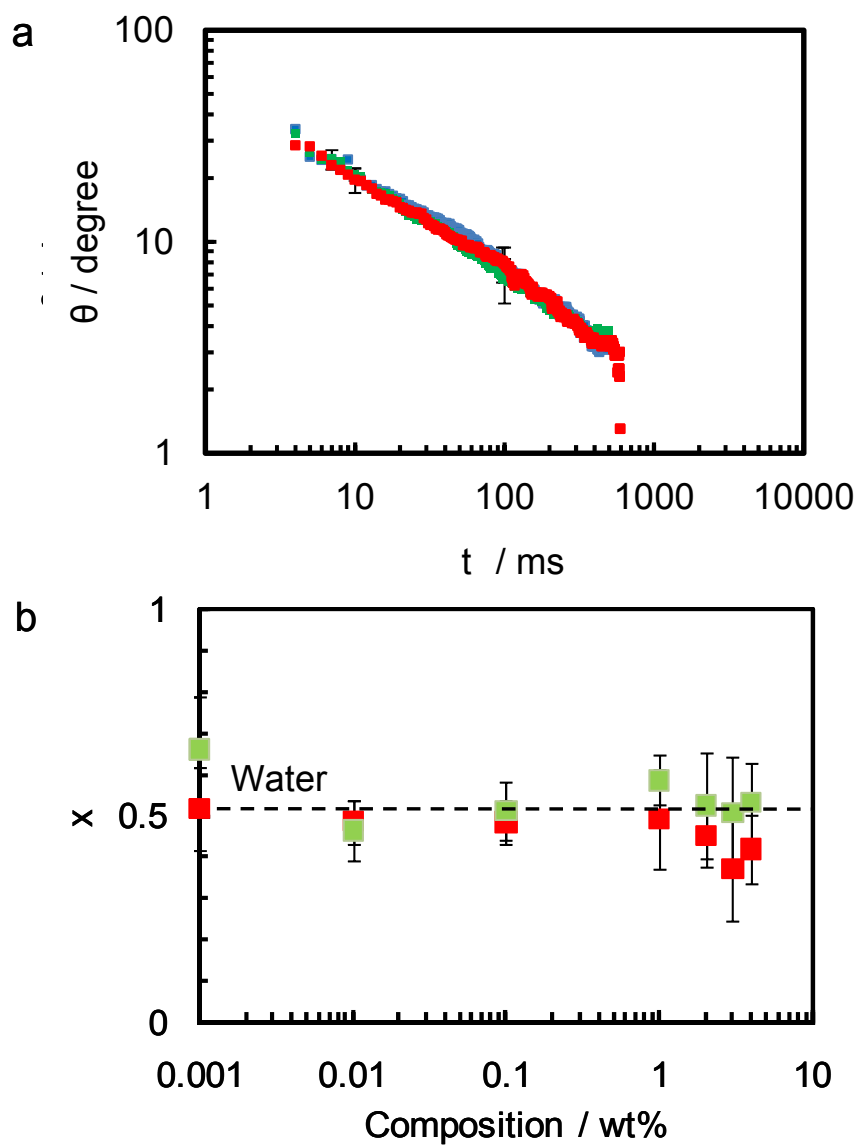

Figure 3

E. Seino et al 

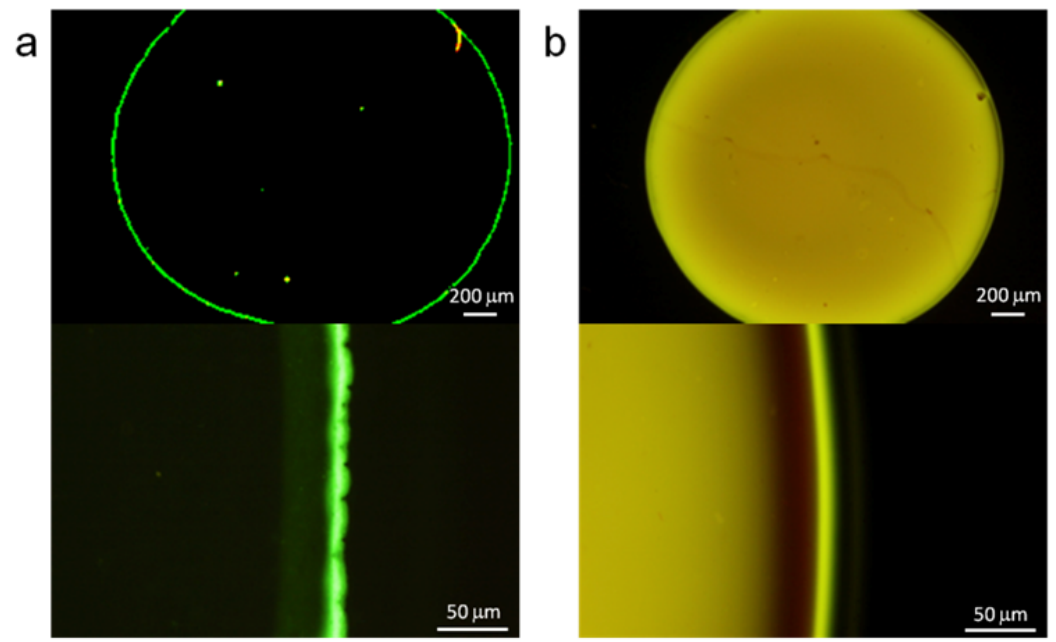

Figure 4

E. Seino et al 


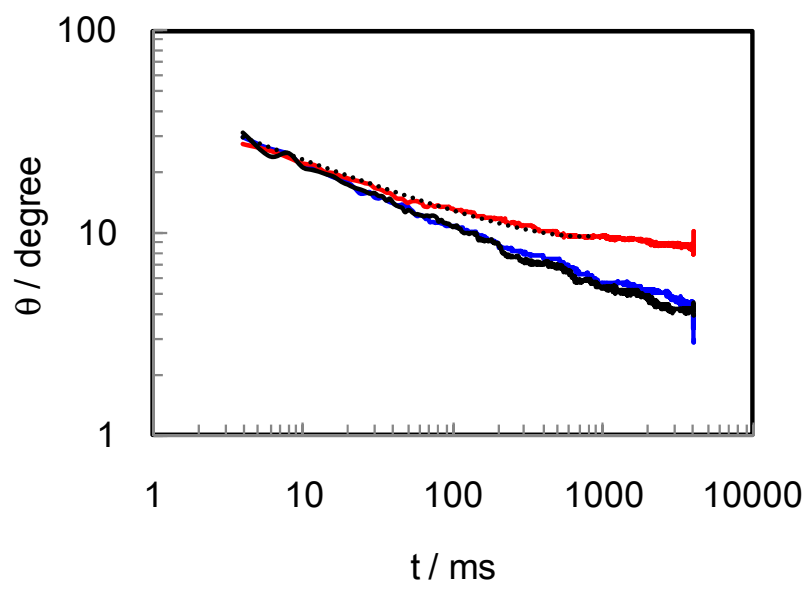

Figure 5

E. Seino et al 

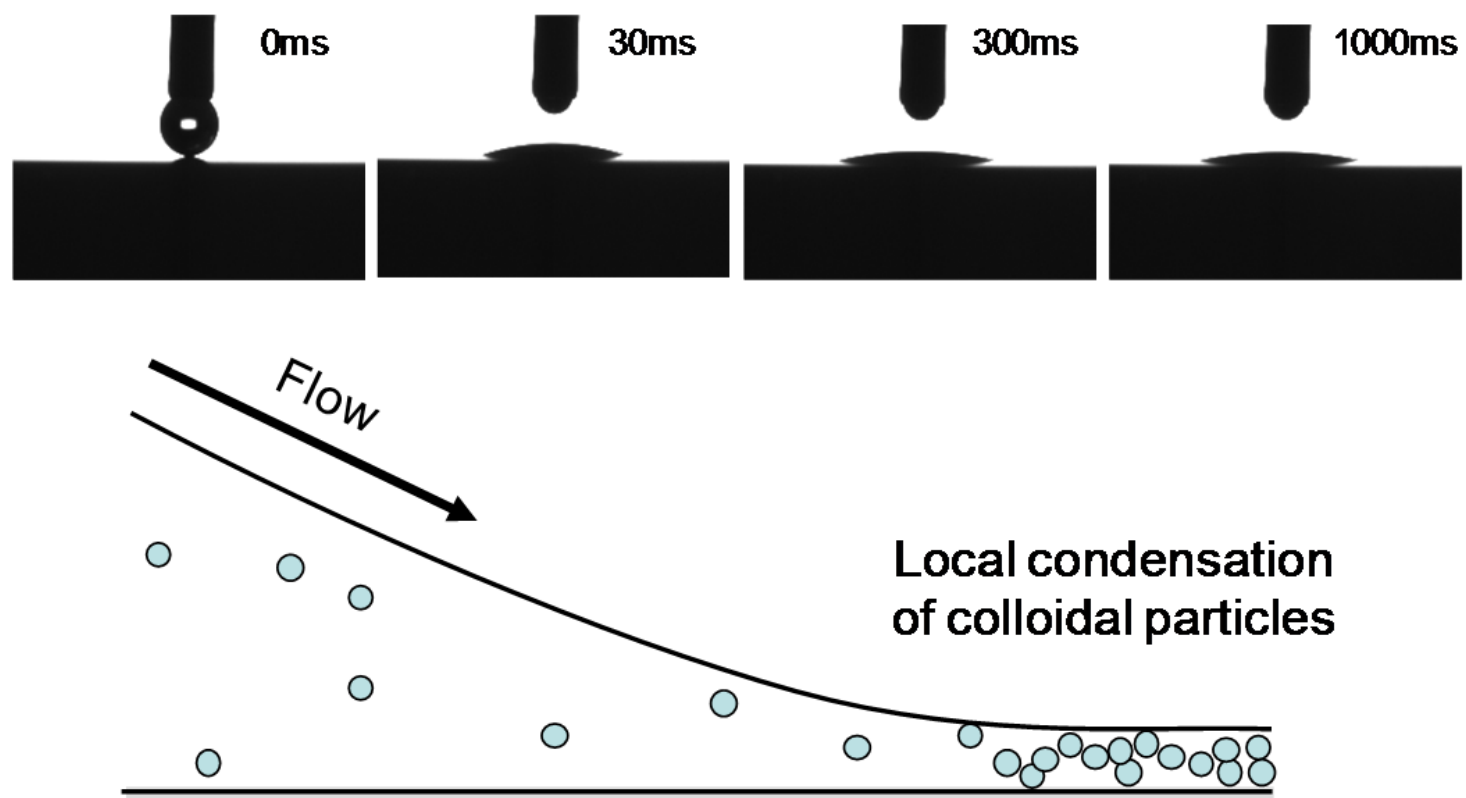

\section{Agar gel}

We have analyzed the effects of the addition of silica particles on the wetting velocity at

flat and fractal agar gel surfaces and the applicability of such particles for controlling the wetting dynamics of water. The wetting is inhibited by the addition of a suitable amount of silica particles with a diameter of $20 \mathrm{~nm}$.

Graphical abstract

E. Seino et al. 


\title{
Wetting dynamics of colloidal dispersions on agar gel surfaces
}

\author{
Eri Seino ${ }^{\dagger}$, Shigeki Chida, ${ }^{\dagger}$ Hiroyuki Mayama ${ }^{\ddagger}$ and Yoshimune Nonomura, ${ }^{\dagger *}$ \\ $\dagger$ \\ Department of Biochemical Engineering, Graduate School of Science and Engineering Yamagata \\ University, 4-3-16 Jonan, Yonezawa 992-8510, Research Institute for Electronic Science, Hokkaido \\ University, CRIS Building, N21W10, Sapporo 001-0021, Japan
}

Accuracy of the contact angle measurements

We estimate that our technique is reliable and accurate when we measure contact angles with a value below $20^{\circ}$. The evidences are as follows:

(1) We measured contact angles of standard samples which were resin plates with liquid droplets drawn on it. The obtained angles were $4.9 \pm 0.0$ and $60.3 \pm 0.0$ degrees when ideal angles were 5.0 and 60.0 degrees, respectively. The average values agree with the setting values of these standard samples and the standard deviations are sufficiently-small.

(2) In the cases of Figure 2 and 3, standard deviations were sufficiently-small values $0.5 \sim 2$ degrees, which are almost similar with the accuracy of the a DM-501 contact angle meter. Some contact angles and their standard deviations are described in Table S1 and S2.

\begin{tabular}{|c|c|c|c|}
\hline \multirow{2}{*}{ Liquid } & \multicolumn{3}{|c|}{ Contact angle (average \pm standard deviation) / degree } \\
\hline & $10 \mathrm{~ms}$ & $100 \mathrm{~ms}$ & $1000 \mathrm{~ms}$ \\
\hline Water & $21.6 \pm 1.8$ & $10.9 \pm 0.5$ & $5.7 \pm 0.7$ \\
\hline $\begin{array}{l}0.1 \text { wt } \% \text { colloidal dispersion } \\
\text { containing silica particles } \\
\text { (diameter }=20 \mathrm{~nm})\end{array}$ & $22.2 \pm 1.4$ & $13.2 \pm 1.7$ & $9.7 \pm 1.4$ \\
\hline $\begin{array}{c}0.1 \mathrm{wt} \% \text { colloidal dispersion } \\
\text { containing silica particles } \\
(\text { diameter }=550 \mathrm{~nm})\end{array}$ & $21.2 \pm 1.3$ & $10.7 \pm 0.8$ & $5.4 \pm 1.0$ \\
\hline \multicolumn{4}{|c|}{ Table S2 Contact angle on fractal agar surface $(n=10)$} \\
\hline \multirow{2}{*}{ Liquid } & \multicolumn{3}{|c|}{ Contact angle (average \pm standard deviation)/ degree } \\
\hline & $10 \mathrm{~ms}$ & $100 \mathrm{~ms}$ & $1000 \mathrm{~ms}$ \\
\hline Water & $19.6 \pm 1.4$ & $6.7 \pm 1.6$ & - \\
\hline $\begin{array}{l}0.1 \mathrm{wt} \% \text { colloidal dispersion } \\
\text { containing silica particles } \\
(\text { diameter }=20 \mathrm{~nm})\end{array}$ & $20.8 \pm 2.6$ & $7.5 \pm 1.5$ & - \\
\hline $\begin{array}{c}0.1 \mathrm{wt} \% \text { colloidal dispersion } \\
\text { containing silica particles } \\
(\text { diameter }=550 \mathrm{~nm})\end{array}$ & $19.6 \pm 2.1$ & $7.9 \pm 0.7$ & - \\
\hline
\end{tabular}

(3) The contact angle $\theta_{\mathrm{D}}$ of water decreased during the spreading process. The pattern of decline was linear on $\log -\log$ plots and followed the power law: $\theta_{\mathrm{D}}$ was proportional to $\mathrm{t}^{-0.27}$ for 0-4000 ms. This result roughly follows Tanner's law for smooth and clean surfaces in which $\theta_{\mathrm{D}}$ is proportional to $t^{-0.3}$. 
Physical properties of silica dispersions

Table S3 Surface tension and viscosity of silica dispersions

\begin{tabular}{cccc}
\hline Particle size / $\mathrm{nm}$ & Composition / wt $\%$ & Surface tension / $\mathrm{mNm}-1$ & Viscosity / cP \\
\hline 20 & 0.001 & $71.3 \pm 0.4$ & $0.89 \pm 0.00$ \\
& 0.01 & $71.2 \pm 0.5$ & $0.88 \pm 0.00$ \\
0.1 & $71.2 \pm 0.3$ & $0.90 \pm 0.01$ \\
& 1 & $70.8 \pm 0.4$ & $1.09 \pm 0.00$ \\
& 2 & $70.3 \pm 0.4$ & $1.39 \pm 0.01$ \\
& 3 & $70.0 \pm 0.2$ & $1.92 \pm 0.00$ \\
& 4 & $69.1 \pm 0.2$ & $2.84 \pm 0.01$ \\
100 & 0.001 & $71.3 \pm 0.2$ & $0.88 \pm 0.00$ \\
& 0.01 & $71.1 \pm 0.1$ & $0.87 \pm 0.01$ \\
& 0.1 & $70.8 \pm 0.4$ & $0.89 \pm 0.02$ \\
& 1 & $70.4 \pm 0.2$ & $0.88 \pm 0.00$ \\
& 2 & $70.3 \pm 0.2$ & $0.89 \pm 0.02$ \\
550 & 3 & $70.1 \pm 0.1$ & $0.89 \pm 0.03$ \\
& 4 & $69.5 \pm 0.2$ & $0.91 \pm 0.01$ \\
& 0.001 & $71.1 \pm 0.4$ & $0.86 \pm 0.01$ \\
& 0.01 & $71.5 \pm 0.3$ & $0.86 \pm 0.02$ \\
& 0.1 & $70.7 \pm 0.2$ & $0.88 \pm 0.01$ \\
1000 & 1 & $70.1 \pm 0.3$ & $0.86 \pm 0.00$ \\
& 2 & $70.6 \pm 0.3$ & $0.89 \pm 0.00$ \\
& 3 & $70.2 \pm 0.3$ & $0.88 \pm 0.01$ \\
& 4 & $69.7 \pm 0.3$ & $0.92 \pm 0.02$ \\
& 0.001 & $71.4 \pm 0.1$ & $0.88 \pm 0.00$ \\
& 0.01 & $71.2 \pm 0.2$ & $0.87 \pm 0.01$ \\
& 0.1 & $70.3 \pm 0.1$ & $0.89 \pm 0.02$ \\
1 & $70.1 \pm 0.4$ & $0.88 \pm 0.00$ \\
2 & $70.0 \pm 0.2$ & $0.89 \pm 0.02$ \\
3 & $70.3 \pm 0.3$ & $0.89 \pm 0.03$ \\
& 4 & & $0.91 \pm 0.01$ \\
\hline
\end{tabular}




\section{Tanner's law}

In this section, we would like to show a process how to derive Tanner's law.

First, we consider dissipation rate of a spreading droplet due to viscosity.

$$
T \dot{S}=\int_{0}^{L} d x \int_{0}^{e} \eta\left(\frac{d v_{x}(z)}{d z}\right)^{2} d z
$$

where $T$ is temperature, $S$ is entropy, $F$ is spreading force applied at contact line, $\eta$ is viscosity of liquid, $V$ is velocity of contact line, $v_{x}(z)$ is velocity along horizontal direction at height $z, L$ is diameter of the droplet and $e$ is the height (thickness) of the spreading droplet as

$$
e=\theta_{D} x
$$

where $\theta_{D}$ is contact angle. The geometry is shown in Figure $\mathrm{S} 1$.

In eq. (S1), $d v_{x}(z) / d z$ can be approximated as

$$
\frac{d v_{x}(z)}{d z} \approx \frac{V}{\theta_{D} x}=\frac{V}{e}
$$

Therefore,

$$
T \dot{S}=\int_{0}^{L} d x \int_{0}^{e} \eta\left(\frac{d v_{x}(z)}{d z}\right)^{2} d z=\int_{0}^{L} d x \eta\left(\frac{V}{e}\right)^{2} e=\frac{\eta V^{2}}{\theta_{D}} \int_{0}^{L} \frac{d x}{x} \approx \frac{\eta V^{2}}{\theta_{D}} \int_{a}^{L} \frac{d x}{x}=\frac{\eta V^{2}}{\theta_{D}} l
$$

where $a$ is the size of a molecule or an atom and $l$ is

$$
l=\int_{a}^{L} \frac{d x}{x}=\ln \frac{L}{a}
$$

On the other hand, $T \dot{S}$ in eq. (S1) can be related to the spreading force applied at contact line $F$ and the velocity of contact line $V$ as

$$
T \dot{S}=F V
$$

Therefore, we get

$$
F=\left(\eta V / \theta_{D}\right) l
$$

It is possible to discuss $F$ from the point of view of the interfacial tensions at the contact line along horizontal direction as

$$
F=\gamma_{L}-\gamma_{L} \cos \theta_{D}
$$

If $\theta_{D} \ll 1, F$ is

$$
F=\left(\theta_{D}^{2} / 2\right) \gamma_{L}
$$

where $\cos \theta \approx 1-\theta^{2} / 2$.

Using eqs. (S7) and (S9), time development of $\theta_{D}$ is discussed. We get

$$
V=\frac{d R}{d t}=\left(\gamma_{L} / 2 \eta l\right) \theta_{D}^{3}
$$

where $R$ is the radius of curvature of the spreading droplet. 
Here, $R$ and $\theta_{D}$ are directly related because the volume of the spreading droplet $\Omega$ is constant as

$$
\Omega=(\pi / 4) R^{3} \theta_{D}
$$

From eq. (S11), we get $V$ to compare to that in eq (S10) as

$$
\frac{d R}{d t}=-\frac{R}{3 \theta_{D}} \frac{d \theta_{D}}{d t}
$$

The time development of $\theta_{D}$ is described by eq. (S12).

The time dependence of $\theta_{D}$ can be determined from eqs. (S10) and (S12).

$$
-\frac{R}{3 \theta_{D}^{4}} \frac{d \theta_{D}}{d t}=\frac{\gamma_{L}}{2 \eta l}
$$

Since the following relation is filled under constant volume

$$
R=\left[4 \Omega /\left(\pi \theta_{D}\right)\right]^{1 / 3}
$$

we get

$$
\begin{gathered}
-\theta_{D}^{-13 / 3} d \theta_{D}=\frac{3 \gamma_{L}}{2 \eta l \Omega^{1 / 3}} d t \\
\theta_{D}^{-10 / 3}=\left(\frac{5 \gamma_{L}}{\eta l \Omega^{1 / 3}}\right) t+C \\
\theta_{D}=\left[\left(\frac{5 \gamma_{L}}{\eta l \Omega^{1 / 3}}\right) t+C\right]^{-3 / 10}
\end{gathered}
$$

Since $\theta_{D} \rightarrow 0$ in superhydrophilic surface when $t \rightarrow \infty, C=0$. Therefore, we obtain Tanner's law as a power law.

$$
\theta_{D} \propto t^{-3 / 10}
$$

Relation to this, the time dependence of $R$ is the following relation by eq. (S14).

$$
R \propto t^{+1 / 10}
$$

The time dependence of the radius on surface $r$ is also

$$
r=R \cos \theta_{D} \propto t^{+1 / 10}
$$




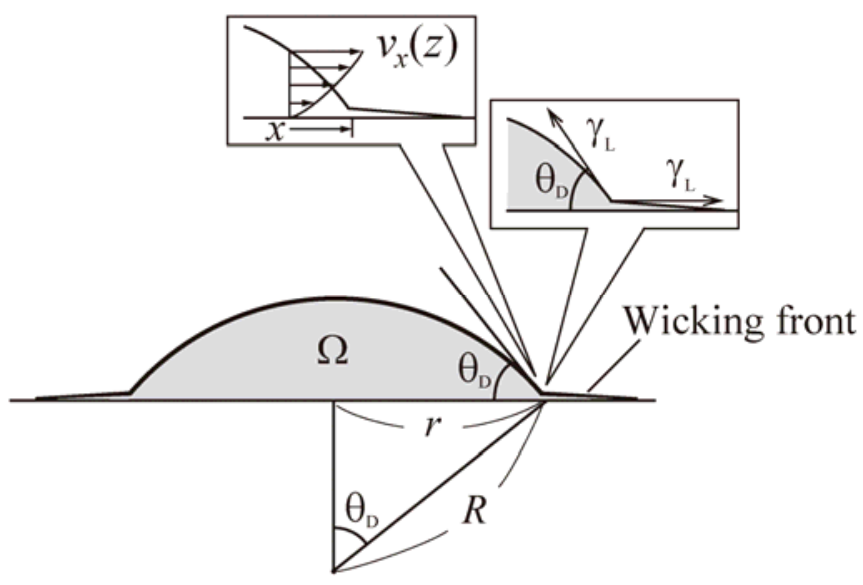

Figure S1. Schematic representation of a spreading droplet. The insets show the distribution of $v_{x}(z)$ and force balance in the vicinity of the contact line.

Reference:

Pierre-Gilles de Gennes, Francoise Brochard-Wyart, David Quere, Capillarity and Wetting Phenomena: Drops, Bubbles, Pearls, Waves (Springer, NY, pp. 141-151 (2003)). 